\title{
Theory of Classical Novae
}

\author{
Mariko Kato*i \\ Keio University \\ E-mail: mariko.kato@st.hc.keio.ac.jp \\ Izumi Hachisu \\ University of Tokyo
}

\begin{abstract}
We briefly review the current understanding of classical novae. Nova light curves basically exhibit a homologous nature, beside the dust blackout and oscillatory behavior, in spite of very different evolution timescale from fast to slow novae. Optically thick winds governs the evolution of decay phase of nova outbursts, of which mass-loss rate depends strongly on the white dwarf (WD) mass and weakly on the chemical composition of ejecta. The optical and near-infrared light curves of novae are reproduced mainly by free-free emission from optically thick winds. Ultraviolet (narrow $1455 \AA$ band) and supersoft X-ray fluxes are reproduced basically by the blackbody emission from the photosphere. These optical, UV, and supersoft X-ray light curves evolve in different timescales, and we can estimate the WD mass from fitting theoretical light curves to observational data. We also discuss the growth rate of the WD via hydrogen/helium burning for various WD masses and accretion rates.
\end{abstract}

The Golden Age of Cataclysmic Variables and Related Objects - III, Golden2015

7-12 September 2015

Palermo, Italy

* Speaker.

${ }^{\dagger}$ A footnote may follow. 


\section{Introduction}

A nova outburst is a thermonuclear runaway event on a white dwarf (WD). The WD accretes hydrogen-rich matter from the companion star. When the accreted matter reaches a critical value, hydrogen nuclear burning becomes unstable that triggers thermonuclear runaway (Kato et al. 2014). The envelope greatly expands and the WD becomes very bright. The optically thick winds begins to blow. After the optical maximum (i.e., maximum expansion of the photosphere), the WD's photosphere moves inward because outward-going ejecta becomes optically thin. Free-free emission from the expanding ejecta dominates the spectrum in the optical and infrared regions. A large part of the hydrogen-rich envelope is blown away.

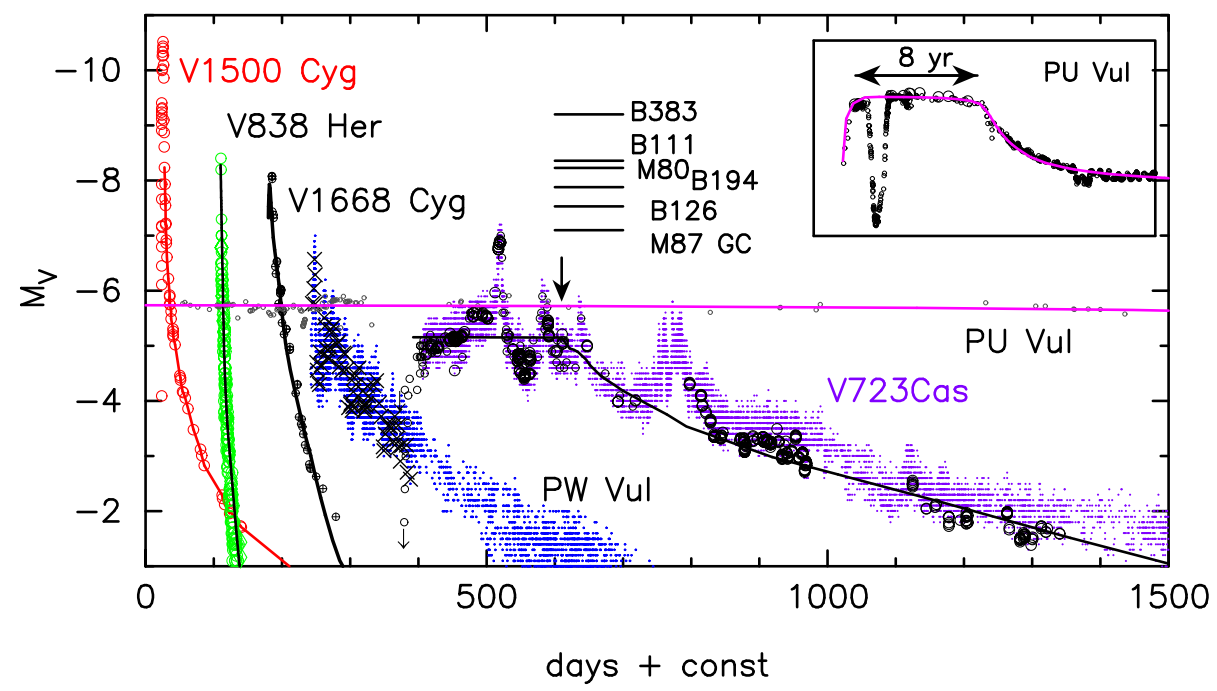

Figure 1: Light curves of various speed classes of novae. From left to right, V1500 Cyg, V838 Her, V1668 Cyg, PW Vul, V723 Cas, and PU Vul (horizontal line and inset). (taken from Kato et al. 2013).

Figure 1 shows $V$ and visual light curves of six well-observed Galactic classical novae with different speed classes. V1500 Cyg is an exceptionally bright (superbright), very fast nova $(\underline{z}=2.9$ days and $t_{3}=3.6$ days). Here, $t_{2}\left(t_{3}\right)$ is the time in days in which a nova decays by two (three) magnitudes from its maximum. V838 Her is a very fast nova, one of the fastest novae except for the superbright novae. It exhibits a normal super-Eddington luminosity in the early phase. V1668 Cyg is a fast nova with $t_{2}=12$ days and $t_{3}=25$ days. PW Vul is a slow nova that exhibits oscillatory behavior in the early phase. V723 Cas is a very slow nova that features multiple peaks in the early stage. PU Vul is a symbiotic nova with a flat optical peak lasting eight years. Its light curve for the first 20 years is plotted in the inset of Figure 1.

Figure 2(a) shows time-normalized light curves of five classical nova with different speed classes. Note that the timescale is normalized to fit the V1500 Cyg data. Figures 2(b) and (c) shows color evolution of these novae. In the early decay phase colors follow that of free-free emission, B$\mathrm{V}=-0.03$, and $\mathrm{U}-\mathrm{V}=-0.97$ (indicated by the horizontal line), until emission lines become dominate in the nebular part. This figure indicates that there is a universal nature in the decay phase of nova light curve among very different speed classes, although the optical (free-free emission) and UV $1455 \AA$ (blackbody emission) emissions originate from different places as shown later. Hachisu and 

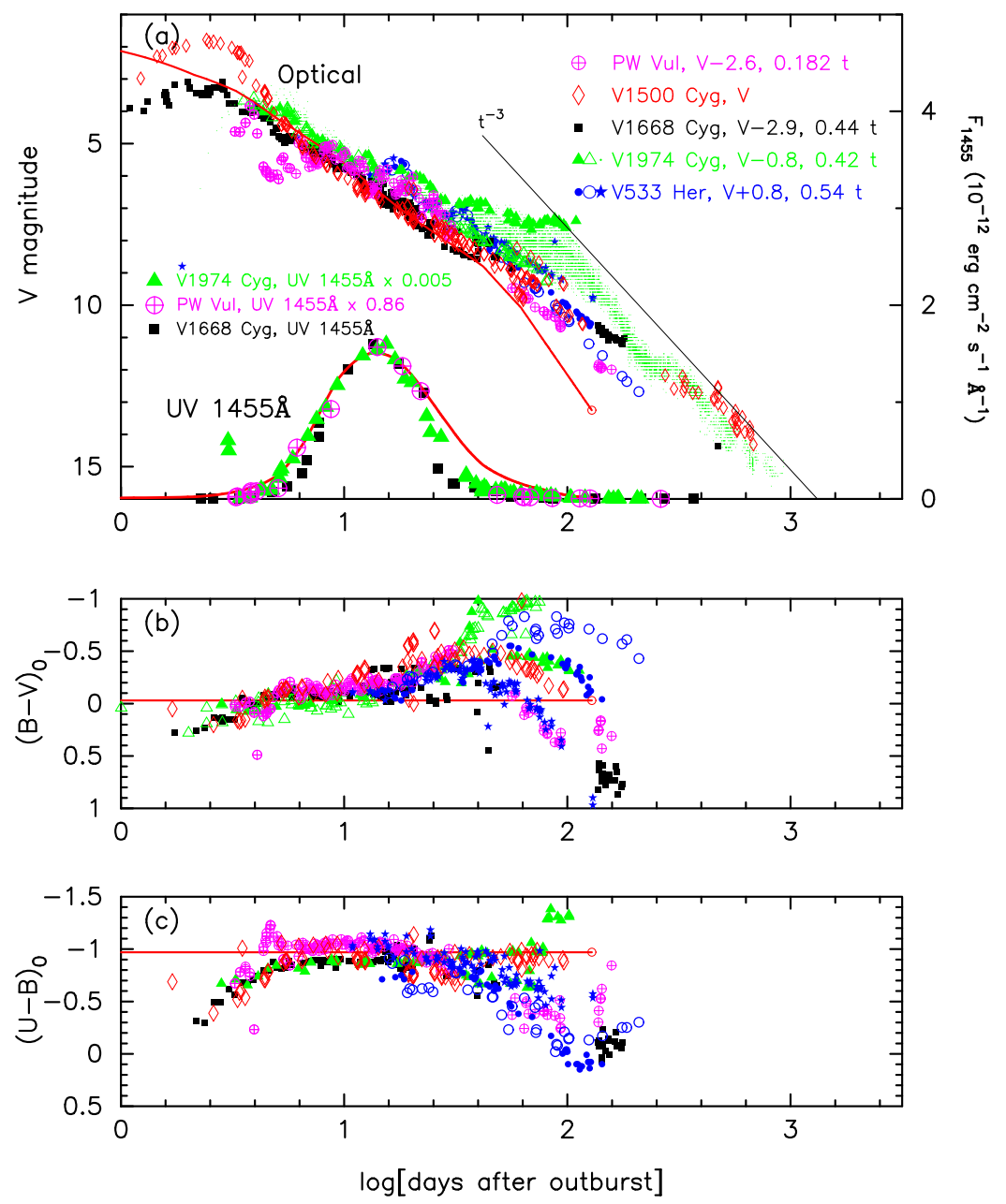

Figure 2: (a) Time-normalized nova light curves of various speed classes. For V-band data, V1500 Cyg, V1668 Cyg, PW Vul, V1974 Cyg, and V533 Her. For UV 1455 A narrow band data, V1974 Cyg, PW Vul, and V1668 Cyg. (b) (B-V) color evolution. (c) (U-B) color evolution. The horizontal line indicates the color of optically thick free-free emission, i.e., $\mathrm{B}-\mathrm{V}=-0.03$, and $\mathrm{U}-\mathrm{V}=-0.97$. The time is normalized to adjust the light curve of V1500 Cyg, and the normalized factor is shown in the figure. (taken from Hachisu and Kato 2015).

Kato (2006) called this property "the universal decline law of classical nova."

\section{Nova light curves}

\subsection{Optically thick wind theory}

It is well known that Henyey-type code, widely used in stellar evolution calculation, has numerical difficulty to calculate the expanding phase of a nova outburst. Kato developed the optically thick wind theory to follow the decay phase of a nova outburst (summary is given in Kato and Hachisu 1994). We solve the equations of motion, mass continuity, radiative diffusion, and conservation of energy, from the bottom of the hydrogen-rich envelope through the photosphere assuming 
a steady-state (Kato \& Hachisu 1994). Decay phases of novae can be followed by a sequence of steady-state and static solutions with the chemical composition of hydrogen-rich envelope.

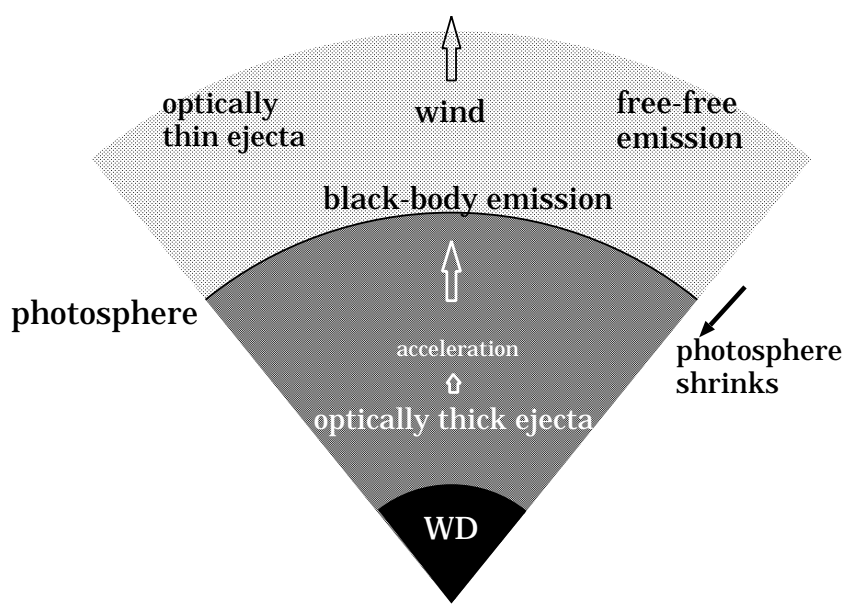

Figure 3: Schematic configuration of emission from nova ejecta. The optically thick winds are accelerated deep inside the photosphere. Optically thin ejecta emits free-free radiation at relatively longer wavelengths while blackbody emission from the photosphere dominates at shorter wavelengths. (Figure taken from Hachisu and Kato, 2006).

Figure 3 illustrate an extended stage of nova outburst. The supersoft X-ray and UV 1445 $\AA$ narrow band fluxes are photospheric origin, so they are calculated from blackbody emission with the photospheric temperature and luminosity. On the other hand, free-free emission from an optically thin plasma contributes to the optical and IR continuum fluxes. The flux of free-free emission is estimated by

$$
F_{V} \propto \int N_{e} N_{i} d V \propto \int_{R_{\mathrm{ph}}}^{\infty} \frac{\dot{M}_{\mathrm{wind}}^{2}}{v_{\mathrm{wind}}^{2} r^{4}} r^{2} d r \propto \frac{\dot{M}_{\mathrm{wind}}^{2}}{v_{\mathrm{ph}}^{2} R_{\mathrm{ph}}},
$$

during the optically thick wind phase, where $F_{v}$ is the flux at frequency $v, N_{e}$ and $N_{i}$ are the number densities of electrons and ions, respectively, $R_{\mathrm{ph}}$ is the photospheric radius, $\dot{M}_{\text {wind }}$ is the wind massloss rate, $v_{\text {ph }}$ is the photospheric velocity, and $N_{e} \propto \rho_{\text {wind }}$ and $N_{i} \propto \rho_{\text {wind }}$. These $\dot{M}_{\text {wind }}, R_{\text {ph }}$, and $v_{\text {ph }}$ values are calculated from our optically thick wind solutions.

\subsection{The universal decline law}

Figure 4 shows light curves calculated for various WD masses. As the wind mass-loss rate quickly decreases with time, the flux also decreases with time. In a more massive WD, the light curve decays more quickly mainly because of its smaller envelope mass (i.e., smaller ignition mass), so the evolution time scale is shorter.

Note that the shape of the light curve is independent of the frequency (or wavelength), whereas the absolute magnitude, i.e., proportionality constant of Equation (2.1), depends on it. This wavelengthfree light curve shape is one of the characteristic properties of free-free emission (see Hachisu \& Kato 2006).

The light curves in Figure 4 shows a remarkable similarity. If we plot these light curves on logarithmic time since the outburst, and shift them in the vertical and horizontal directions (i.e., 


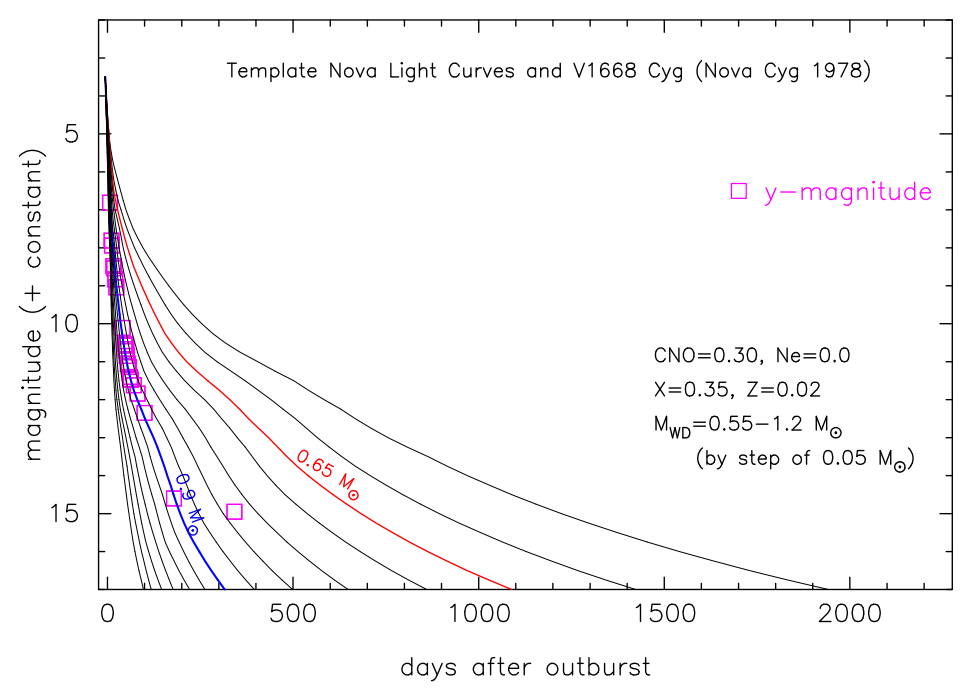

Figure 4: Free-free emission model light curves for various WD masses from $0.55 M_{\odot}$ to $1.2 M_{\odot}$. Open squares denote observational $y$ magnitudes (moderately narrow optical band, that represent almost line-free magnitude) of V1668 Cyg that show good agreement with the $0.9 M_{\odot}$ model (Figure taken from Hachisu \& Kato 2010).

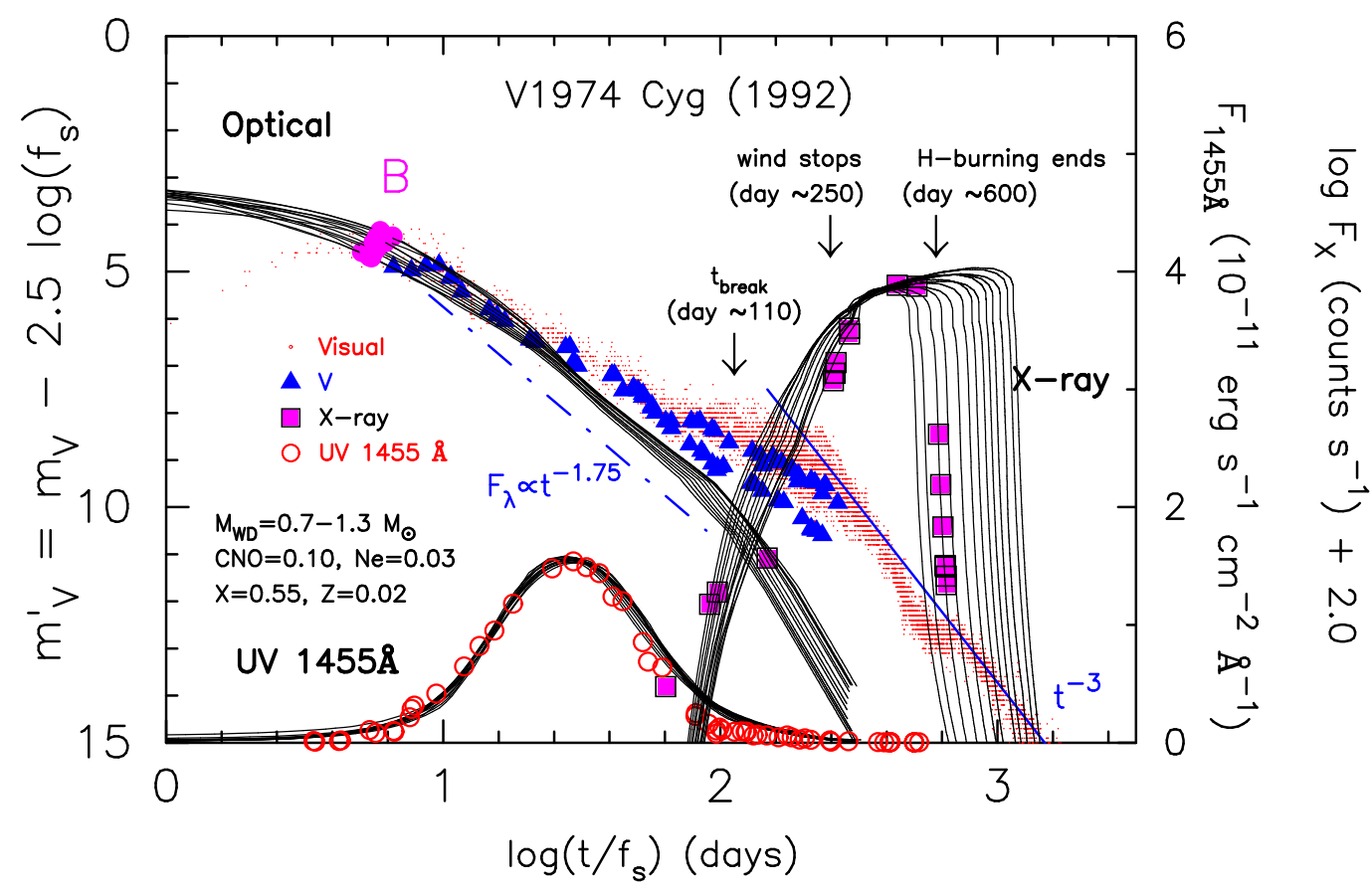

Figure 5: Free-free emission model light curves for various WD masses from $0.7 M_{\odot}$ to $1.3 M_{\odot}$ by steps of $0.05 M_{\odot}$. The time scales are squeezed or stretched by a factor of $f_{\mathrm{s}}$ along time to fit each other and the magnitude is shifted by $-2.5 \log f_{\mathrm{s}}$. The two straight lines indicate the decline rates of free-free flux, i.e., $F_{V} \propto t^{-1.75}$ (dash-dotted line) and $F_{V} \propto t^{-3}$ (solid line). Observational data of V1974 Cyg are superposed (Figure taken from Hachisu \& Kato 2010 ). 
normalize them in the time direction by a factor of $f_{\mathrm{s}}$ and also shift the magnitude in the vertical direction by $-2.5 \log f_{\mathrm{s}}$ ), all the light curves essentially converge into a single curve independently of the WD mass and chemical composition as demonstrated in Figure 5 (Hachisu \& Kato 2010). Figure 5 also shows the UV $1455 \AA$ narrowband light curve. This narrow band is defined observationally to avoid prominent emission and absorption lines in the nova UV spectra and to represent the continuum flux (Cassatella et al. 2002). We see that the model light curves of the UV $1455 \AA$ band also converge into a single curve by using the same time-scaling factor of $f$. In our model light curves, X-ray turnoff times do not converge, because the supersoft X-ray phase (duration of steady nuclear burning) depends on the hydrogen burning rate, which does not follow the timesscaling law.

Figure 5 also shows the characteristic properties of nova evolution. After the optical maximum (typically around point B), the photospheric temperature rises with time, and the main emitting wavelength region shifts from optical to UV and then to supersoft X-ray. The optically thick winds continue until the photospheric temperature reaches $\log T(\mathrm{~K})>5.2$ (which corresponds to the opacity peak from iron ionization). The WD photosphere emits supersoft X-rays until hydrogen nuclear burning stops. After the hydrogen burning is extinguished, the nova enters a cooling phase and finally the WD becomes dark.

\subsection{MMRD relation}

Using this universality of nova light curves, we can derive a theoretical maximum magnitude versus rate of decline for novae (the MMRD relation). The idea is to solve the degeneracy of peak magnitude in Figure 5 for various WD masses as a function of $f_{s}$ (Hachisu and Kato 2010). This theoretical MMRD relation agrees well to those observationally derived and thus, provides theoretical explanation of the nova speed class. The figures include theoretically and observationally derived MMRD relations as well as comparison with individual nova data (Figures 31 and 36 in Hachisu and Kato 2015, also Figures 47-49 in Hachisu and Kato 2016). Note that recurrent novae do not follow the MMRD relations, and locate much below the MMRD relations because of their small envelope mass (see above references).

\subsection{WD mass estimated by light curve fittings}

Figure 6 shows the same light curves as in Figure 5 but on a real time scale and in the absolute $V$ magnitude. In a light curve analysis, we can select the best-fit theoretical model by comparing observed data with models, but only the optical light curve is not enough. This is because the main body of the optical light curve follows the same decline rate of $t^{-1.75}$ and we can fit several light curves of different WD masses with the optical data as seen in Figure 6. If we have information other than optical, such as the UV $1455 \AA$ flux or X-ray turn-on and turnoff times, we can accurately select one model light curve. This is why multiwavelength observations are important for determination of the nova parameters.

Figures 5 and 6 also show light curve data of the classical nova V1974 Cyg. The optical light curve decays as $t^{-1.75}$ from the optical peak at $t=2.67$ days (point B). There are two different sets of $V$-magnitudes, the difference becomes remarkably in the nebular phase. This difference is owing to use of different $V$ filters that respond contamination of strong emission lines such as [O 


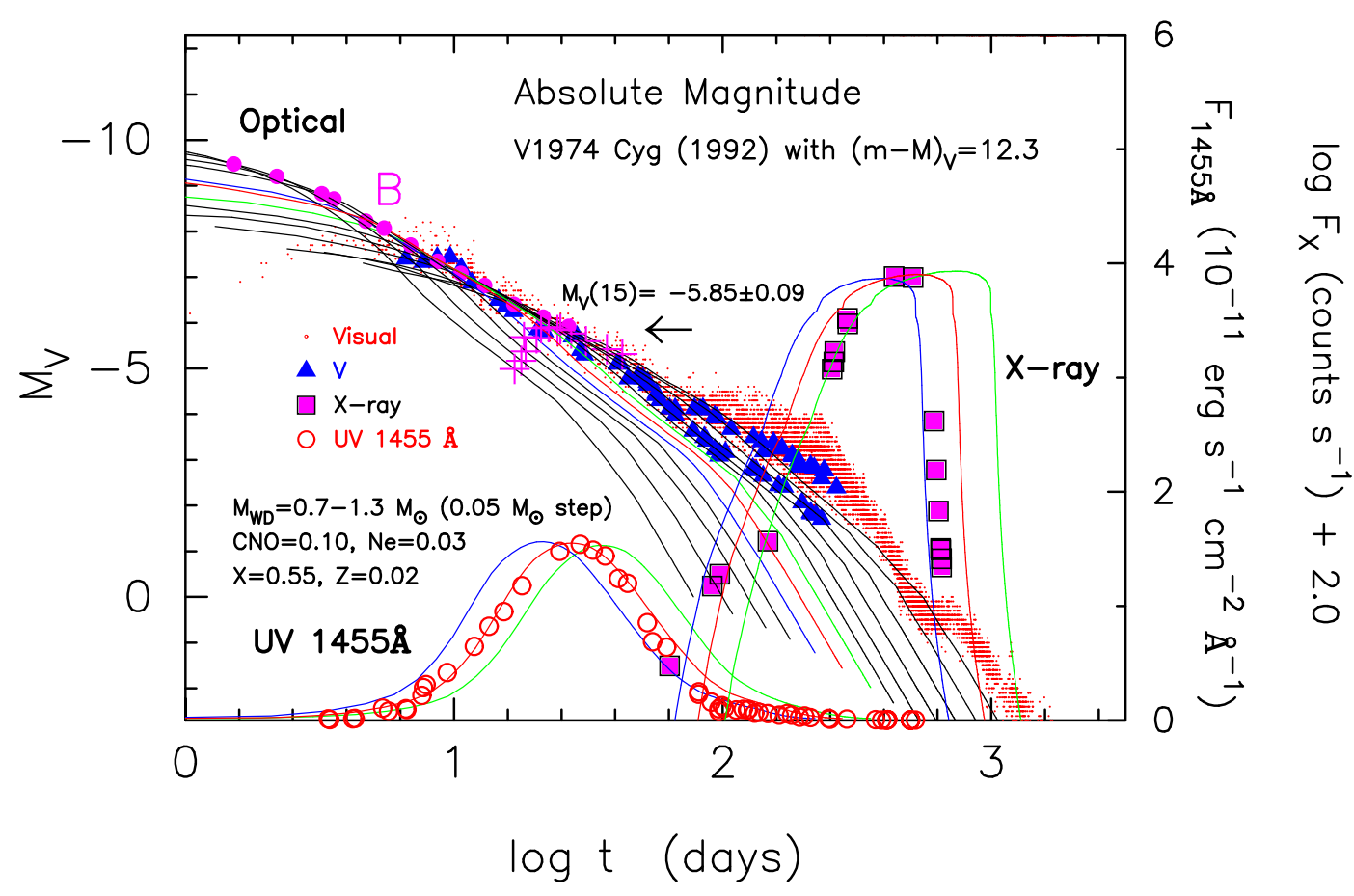

Figure 6: Same as Figure 3 but on a real time scale and in the absolute $V$ magnitude (taken from Hachisu $\&$ Kato 2010). Model UV and supersoft X-ray light curves are plotted only for three WD masses $\left(1.1 M_{\odot}\right.$, $1.05 M_{\odot}$, and $1.0 M_{\odot}$ ) to improve readability.

III] differently in the edge of the $V$ band. The theoretical free-free flux mimics the continuum flux. After the optically-thick winds stops, the magnitude decays as $t^{-3}$, which represents free-expansion of the ejecta.

Because the optical, UV, and supersoft X-ray light curves have different dependencies on the WD mass, we can choose a best-fit model that reproduces these three light curves simultaneously as shown in Figure 6. In this way, we have estimated the WD mass in many classical novae, recurrent novae, and a symbiotic nova. For example, $1.20 M_{\odot}$ for V1500 Cyg and $1.05 M_{\odot}$ for GK Per (Hachisu \& Kato 2014), $0.83 M_{\odot}$ for PW Vul, $0.78 M_{\odot}$ for V705 Cas, $0.65-0.75 M_{\odot}$ for GQ Mus, $0.5-0.60 M_{\odot}$ for RR Pic, $0.5-0.55 M_{\odot}$ for V5558 Sgr, HR Del and V723 Cas (Hachisu \& Kato 2015), $0.98 M_{\odot}$ for V1668 Cyg, $0.98 M_{\odot}$ for V1974 Cyg, $0.96 M_{\odot}$ for QU Vul, $1.0 M_{\odot}$ for V351 Pup, $1.23 M_{\odot}$ for V382 Vel, and $1.15 M_{\odot}$ for V693 CrA for Ne nova (Hachisu \& Kato 2016).

\subsection{Very slow novae with a flat optical peak}

PU Vul is a symbiotic nova that exhibits a long-lasting flat optical peak followed by a slow decline (see Figure 1). During the flat optical peak, the spectral features resemble to F/A supergiants and no massive winds are suggested. Such an evolution can be understood as the case in which no optically thick winds are accelerated and its evolution is followed by a quasi-evolution model of an outburst on a $\sim 0.6 M_{\odot}$ WD (Kato et al. 2011, 2012) (see the purple line in Figure 1).

In majority of classical novae (that occur on a WD of $M_{\mathrm{WD}}>0.7 M_{\odot}$ ) the strong optically thick winds are accelerated and carry most of the envelope mass in a short time. As a result, 
their evolutions are fast and exhibit a sharp optical maximum. In contrast, in less massive WDs ( $\leq 0.6 M_{\odot}$, which depends slightly on the chemical composition) the optically thick winds are not accelerated and thus the nova evolution is extremely slow (as in Figure 1). If the WD mass is less massive than that in PU Vul, the outburst should be much slower and the flat peak lasts much longer than 8 years. In such a case, we may classify it as a F or A type supergiant, and may not recognize it as a nova outburst. This is one of the reason that mean WD mass of classical nova $(\sim 1.0 \mathrm{M}$. $)$ is larger than the field WD or central stars of planetary nebulae $(\sim 0.6 M$.$) .$

The very slow novae V723 Cas, HR Del, and V5558 Sgr exhibit a flat pre-maximum phase with no indication of a strong wind-mass loss, followed by a smooth decline with massive winds after some oscillatory phase of the optical light curves. This sequence can be understood as a transition from initial quasi-static evolution to optically thick wind evolution. The oscillatory behaviors in the light curves may correspond to a relaxation process associated with the transition from static to wind solutions (Kato and Hachisu 2011).

\section{WD mass increases or decreases}

\subsection{Chemical composition of ejecta}

In long term evolution of novae, mass-accreting WDs grow their masses in some cases and decrease in other cases. One of the observational clues for this issue is chemical composition of ejecta in nova outbursts. The IUE satellite observed many nova outburst and showed that nova spectra are enriched by emission lines of heavy elements like C, $\mathrm{O}$ and $\mathrm{Ne}$ (e.g., http://archive.stsci.edu/iue/). These heavy element enrichment in nova ejecta amounts a few to several tens of percent by mass (see Table 1 in Hachisu \& Kato 2006 for a summary). This enhancement is considered to be WD origin (e.g., Prialnik 1986). As the surface of a WD core is eroded during nova outbursts and blown off in the nova wind, the WD mass decreases after each nova cycle.

On the other hand, in recurrent novae, the composition of ejecta is very different from those of classical novae and is almost solar. Theoretical calculations showed that a certain amount of processed helium is added to the WD after every outburst (Prialnik \& Kovetz 1995). The WD could then experience repeated weak helium shell flashes. The resultant wind is weak, and a large part of the envelope mass remains on the WD (Kato \& Hachisu 2004). Therefore, the WD increases its mass in recurrent novae such as U Sco and RS Oph.

\subsection{Mass decreasing WDs}

Hachisu and his collaborators have determined the WD masses for a number of novae from light curve fitting. Figure 7 shows an example of light curve fitting: the neon nova QU Vul (Nova Vul 1984 No.2). As already explained, the theoretical light curve depends strongly on the WD mass and weakly on the chemical composition of ejecta. Hachisu and Kato (2016) calculated various sets of these parameters and choose a $0.96 M_{\odot}$ WD (Figure $7 \mathrm{a}$ ). Figure $7 \mathrm{~b}$ present the total $V$ flux that includes contribution of blackbody emission from the photosphere in addition to free-free emission from the outside of the photosphere to confirm the $0.96 M_{\odot}$ model reproduces both the observed optical and UV light curve data.

QU Vul is a neon nova that indicates the WD consisting of ONeMg WD. Stellar evolution calculation shows that a natal WD, just born from the red giant core, has a mass $>1.07 \mathrm{M}$. . This 

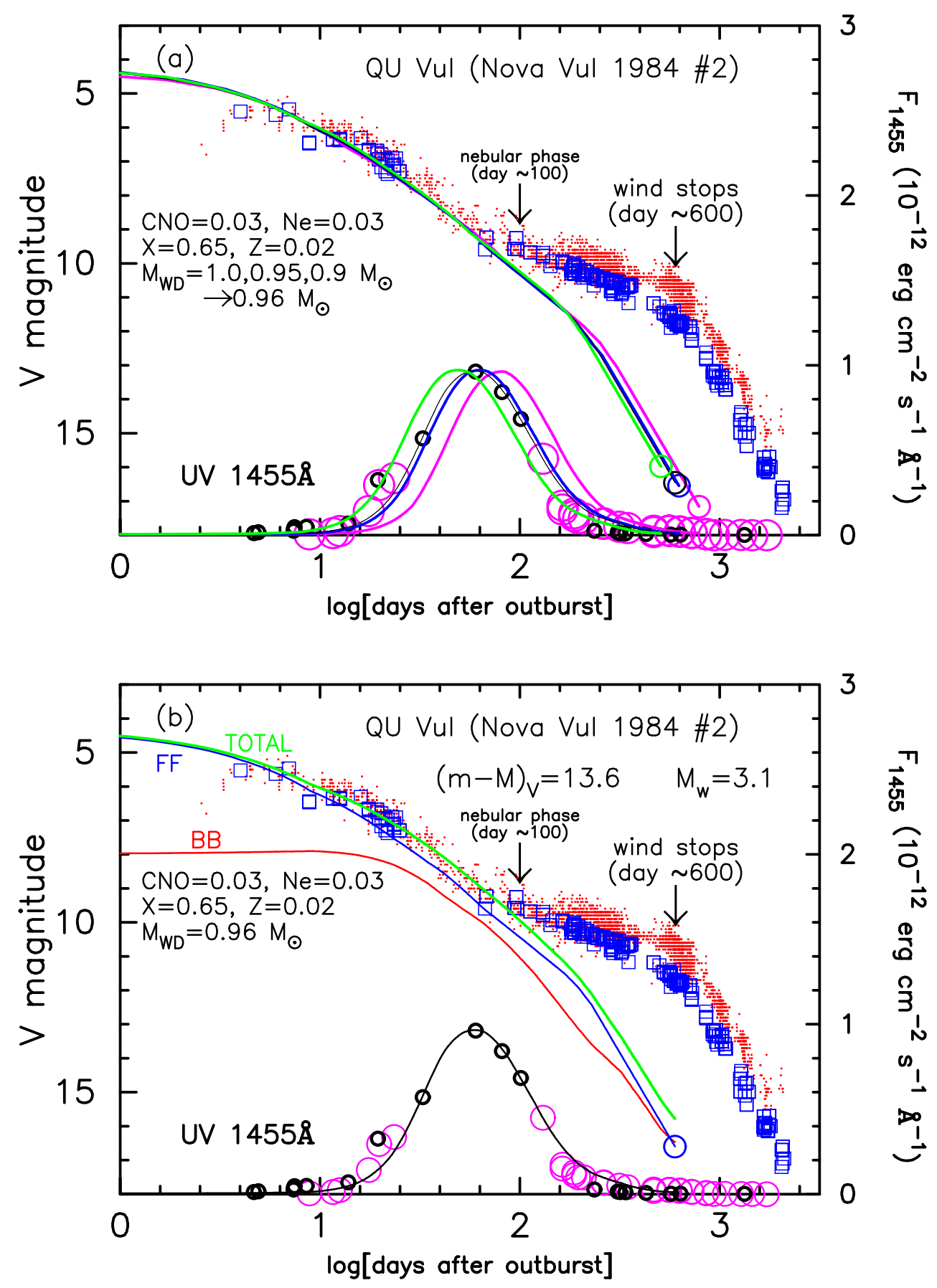

Figure 7: Light curve fitting for QU Vul (Nova Vul 1984 No.2) with Optical and UV $1455 \AA$ data. (a)Solid line denote the theoretical free-free and UV light curve for the WD mass of $0.9,0.95$, and $1.0 M_{\odot}$. (b) Theoretical curves for $0.95 M_{\odot}$ WD. The optical curves are the summation of free-free (blue) and blackbody emission (red).(taken from Hachisu \& Kato 2016). 
means that the WD in QU Vul has lost its mass at least 0.1 $M_{\odot}$ since its birth (Hachisu and Kato 2016). If we assume the ejected mass during one nova outburst to be $\sim 10^{-5} M_{\odot}$, the number of nova outburst is calculated to be about $0.1 M_{\odot} / 10^{-5} M_{\odot}=10,000$ outbursts. We conclude that the WD in QU Vul has lost its mass $\left(>0.1 M_{\odot}\right)$ through such a large number of outburst.

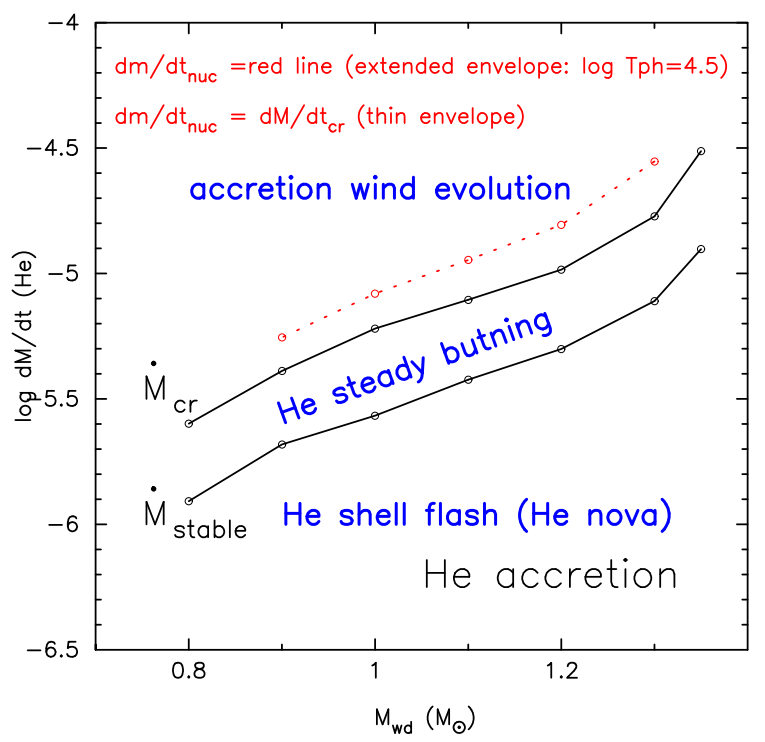

Figure 8: Responce of WDs to mass accretion in the WD mass and the He mass-accretion rate plane. For the accretion rate above the line $\dot{M}_{\mathrm{cr}}$, the helium burning is stable but a part of the envelope will be lost in the wind. For the accretion rate between $\dot{M}_{\text {cr }}$ and $\dot{M}_{\text {stable }}$, the helium accretion is stable and the binary undergoes steady state evolution. Below the line of $\dot{M}_{\text {stable }}$, helium shell burning is unstable and helium nova occurs.

\subsection{Mass increasing WD}

In recurrent novae, the WD is considered to increase. The reason is: (1) The solar composition ejecta indicates no mixing of WD material, so the WD surface is not eroded during nova outbursts. (2) Diffusion process of elements may not effective for short recurrence periods. (3) Outbursts in recurrent novae are weak and certain amount of accreted matter remains on the WD after every outburst (e.g. Prialnik and Kovetz 1995).

Whether the WD mass increases or not is an important factor in binary evolution scenarios, so we summarize here the mass accumulation efficiency of hydrogen and helium burning based on the optically thick wind theory. The efficiency depends on the WD mass and the mass accretion rate.

For hydrogen shell flashes, the first formulation of mass accumulation efficiency appeared in Hachisu et al. (1999) (Equation (9)). There are two boundaries that divide three regions of unstable shell flash (nova outburst region), steady-state burning, and accretion-wind evolution. These boundaries are recently updated by Kato et al. (2014). (see Equations (4) and (5) for $\dot{M}_{\text {stable }}$ and $\dot{M}_{\text {cr }}$ ). Under the lower boundary, i.e., between nova and steady state burning, (i.e., RN region ) they assumed a sudden change of mass accumulation efficiency, i.e., a step function from 0 to 1 , that means a gap between the recurrent nova region (efficiency 0 ) and steady burning region (efficiency 1.0). This approximation is not good and should be updated. 
In mass increasing WDs, the ash helium accumulates underneath hydrogen burning zone, and when the amount of helium reaches the critical value, helium shell flash suddenly occurs. The accumulation efficiency of such helium shell flash is studied by Kato \& Hachisu (2004) who formulated it as their Equations (1)-(6). Figure 8 shows the stability line $\dot{M}_{\text {stable }}$ and $\dot{M}_{\text {cr }}$ based on Figure 1 in Kato \& Hachisu (2004).

As shown in this figure, helium burning is stable for the helium mass accretion rate between $\dot{M}_{\text {cr }}$ and $\dot{M}_{\text {stable }}$ and the binary undergoes steady state evolution in which helium burns at the same rate as the accretion. Above the $\dot{M}_{\text {cr }}$ line, He burning is stable, but the WD loses excess accreted matter, $\dot{M}_{\text {acc }}-\dot{M}_{\text {cr }}$, by winds. As the accretion rate increases, the photospheric radius increases and the wind mass loss rate increases (see Figure 1 of Kato \& Hachisu 2004). The nuclear burning rate (steady state) also increases in the above region $\left(>\dot{M}_{\mathrm{cr}}\right)$, to drive the more massive winds. Note that $d M / d t_{\text {nuc }}$ weakly depends the envelope radius. In an extended envelope (e.g., $\left.\log T_{\mathrm{ph}}(\mathrm{K})=4.5\right)$ it is slightly larger (red dotted line) than plane parallel envelope $\left(\dot{M}_{\mathrm{nuc}}=\dot{M}_{\mathrm{cr}}\right)$.

\section{References}

[1] Cassatella, A., Altamore, A., González-Riestra, R., A\&Ap, 2002, 384, 1023

[2] Hachisu, I., Kato, M., ApJS, 2006, 167, 59

[3] I.Hachisu, \& M.Kato, ApJ, 2014, 785:97

[4] I.Hachisu, \& M.Kato, ApJ, 2015, 798:76

[5] I.Hachisu, \& M.Kato, ApJ, 2016,in press

[6] Hachisu et al., 1999, ApJ, 519,314

[7] Y.Hillman, D.Prialnik, A.Kovetz, M.M.Shara \& D. Neill, 2014, MMRAS,437,1962

[8] Kato, M., \& Hachisu, I.: 1994, ApJ 437, 802

[9] Kato, M., Hachisu, I.: 2004, ApJ, 613, L129

[10] Kato, M., \& Hachisu, I. ApJ, 2011, 743, 157

[11] M. Kato, I. Hachisu, A. Cassatella, R. Gonzalez-Riestra, ApJ,2011, 727, 72

[12] M.Kato, I. Hachisu, \& Henze, M.: 2013, ApJ, 779, 19

[13] M. Kato, J. Mikołajewska, \& I. Hachisu, ApJ,(2012) 750: 5

[14] M. Kato, H. Saio, I. Hachisu, \& K. Nomoto, ApJ,(2014), 793:136

[15] A.Kovetz, ApJ, (1998), 495:401

[16] D.Prialnik, ApJ,(1986), 310, 222

[17] Prialnik, D., Kovetz, A.: 1995, ApJ 445, 789

\section{DISCUSSION}

GIORA SHAVIV's Comment: Please note that Kovetz published a paper in which he explains the treatment of the surface, how the optically thick wind is treated, and how in previous models the mass loss was treated. 
KATO: As frequently discussed in this meeting, the boundary condition is very important. In nova case, the surface boundary conditions are crucially affects the light curve because it determines the photospheric temperature, luminosity, and radius as well as the wind mass loss rate. As commented in my talk, no papers on time-dependent calculation of nova describe on the surface boundary condition in detail. Your mentioned paper by Kovetz (1998) is rare exception that describes the treatment of the surface in detail as you said, however, no sentences were given where the author set the boundary between the optically thick wind and interior solution. It crucially affects the results. For example, Hillman et al. (2014) presented rectangular shape of nova light curves which does not resemble real novae. However, no description is given for surface boundary conditions so I cannot understand what they did. 